\title{
MULTIDIMENSIONAL SELF-AFFINE SETS: NON-EMPTY INTERIOR AND THE SET OF UNIQUENESS
}

\author{
KEVIN G. HARE AND NIKITA SIDOROV
}

\begin{abstract}
Let $M$ be a $d \times d$ real contracting matrix. In this paper we consider the self-affine iterated function system $\{M v-u, M v+u\}$, where $u$ is a cyclic vector. Our main result is as follows: if $|\operatorname{det} M| \geq 2^{-1 / d}$, then the attractor $A_{M}$ has non-empty interior.

We also consider the set $\mathcal{U}_{M}$ of points in $A_{M}$ which have a unique address. We show that unless $M$ belongs to a very special (non-generic) class, the Hausdorff dimension of $\mathcal{U}_{M}$ is positive. For this special class the full description of $\mathcal{U}_{M}$ is given as well.

This paper continues our work begun in [5, 6].
\end{abstract}

\section{NON-EMPTY INTERIOR}

Let $d \geq 2$ and $M$ be a $d \times d$ real matrix whose eigenvalues are all less than 1 in modulus. Denote by $A_{M}$ the attractor for the contracting self-affine iterated function system (IFS) $\{M v-u, M v+u\}$, i.e., $A_{M}=\left\{\pi_{M}\left(a_{0} a_{1} \ldots\right) \mid\right.$ $\left.a_{n} \in\{ \pm 1\}\right\}$, where

$$
\pi_{M}\left(a_{0} a_{1} \ldots\right)=\sum_{k=0}^{\infty} a_{k} M^{k} u .
$$

If $A_{M} \ni x=\pi_{M}\left(a_{0} a_{1} \ldots\right)$, then we call the sequence $a_{0} a_{1} \cdots \in\{ \pm 1\}^{\mathbb{N}}$ an address of $x$. We assume our IFS to be non-degenerate, i.e., $A_{M}$ does not lie in any $(d-1)$-dimensional subspace of $\mathbb{R}^{d}$ (i.e., $A_{M}$ spans $\mathbb{R}^{d}$ ). Let $u \in \mathbb{R}^{d}$ be a cyclic vector for $M$, i.e., $\operatorname{span}\left\{M^{n} u \mid n \geq 0\right\}=\mathbb{R}^{d}$.

Our main result is as follows.

Theorem 1.1. If

$$
|\operatorname{det} M| \geq 2^{-1 / d}
$$

then the attractor $A_{M}$ has non-empty interior. In particular, this is the case when each eigenvalue of $M$ is greater than $2^{-1 / d^{2}}$ in modulus.

Remark 1.2. Note that if $|\operatorname{det} M|<\frac{1}{2}$, then $A_{M}$ is a null set (see [4]) and therefore, has empty interior. It is an interesting question whether $2^{-1 / d}$ in Theorem 1.1 can be replaced with a constant independent of $d$.

2010 Mathematics Subject Classification. 28A80.

Key words and phrases. Iterated function system, self-affine set, set of uniqueness.

Research of K. G. Hare was supported by NSERC Grant RGPIN-2014-03154. 
Corollary 1.3. For an IFS $\left\{M v+u_{j}\right\}_{j=1}^{m}$ with $m \geq 2$ the same claim holds, provided the IFS is non-degenerate.

Proof. Clearly, the attractors are nested as $m$ increases, so it suffices to establish the claim for $m=2$. This, in turn, follows from Theorem 1.1 via an affine change of coordinates.

The history of the problem is as follows. In [1] it was shown that for $M=\left(\begin{array}{cc}\lambda & 0 \\ 0 & \mu\end{array}\right)$, if $0.953<\lambda<\mu<1$, then $(0,0)$ has a neighbourhood which lies in $A_{M}$. Their method was a modification of the one suggested in [4]. In [5] we improved their lower bound to 0.83 . In [6] we proved analogous results for all $2 \times 2$ matrices $M$ by using a similar approach as in [5] for the matrices with real eigenvalues and a different one for the rest. This second approach is the one we use in the current paper.

To prove Theorem 1.1, we need some auxiliary results. These are natural generalizations of those from [6, Appendix] whose proofs had been provided by V. Kleptsyn [7]. We use + for the Minkowski sum of two sets:

$$
A+B=\{a+b \mid a \in A, b \in B\} .
$$

Lemma 1.4. Let $\gamma$ be a path in $\mathbb{R}^{n}$. Let $\gamma^{\prime}\left(t_{1}, t_{2}, \cdots, t_{n-1}\right)=\gamma_{1}\left(t_{1}\right)+$ $\cdots+\gamma_{n-1}\left(t_{n-1}\right)$ where the $\gamma_{i}$ are paths in $\mathbb{R}^{n}$. Let $\delta$ be the diameter of $\gamma\left(\left[s_{1}, s_{2}\right]\right)$, and assume that there is no point in the interior of the surface $\sigma=\left\{\gamma(s)+\gamma^{\prime}(t): s, t \in \partial\left(\left[s_{1}, s_{2}\right] \times[0,1]^{n-1}\right)\right\}$. Then the sets $\gamma\left(s_{1}\right)+$ $\gamma^{\prime}\left([0,1]^{n-1}\right)$ and $\gamma\left(s_{2}\right)+\gamma^{\prime}\left([0,1]^{n-1}\right)$ coincide outside a $\delta$-neighbourhood of $\gamma\left(\left[s_{1}, s_{2}\right]\right)+\gamma^{\prime}\left(\partial\left([0,1]^{n-1}\right)\right)$.

Proof. Assume the contrary and let $z$ be a point of the surface $\widetilde{\gamma}:=\gamma\left(s_{1}\right)+$ $\gamma\left(t_{1}\right)$ (for some $t_{1} \in[0,1]^{n-1}$ ) that lies outside the $\delta$-neighbourhood and that does not belong to the surface $\gamma\left(s_{2}\right)+\gamma\left([0,1]^{n-1}\right)$. By continuity, there is a $\varepsilon$-neighbourhood of $z$ that the latter surface does not intersect.

Now, by the Jordan-Brouwer separation theorem, in this neighbourhood one can find two points "on different sides" with respect to $\widetilde{\gamma}$.

This implies that one of these two points is in the interior of $\sigma=\{\gamma(s)+$ $\left.\gamma^{\prime}(t): s, t \in \partial\left(\left[s_{1}, s_{2}\right] \times[0,1]^{n-1}\right)\right\}$.

Proposition 1.5. If $\gamma_{1}, \gamma_{2}, \cdots, \gamma_{n}$ be $n$ paths in $\mathbb{R}^{n}$ whose span is $\mathbb{R}^{n}$, then $\gamma_{1}+\gamma_{2}+\cdots+\gamma_{n}$ has non-empty interior.

Proof. Let $t=\left(t_{2}, t_{3}, \ldots, t_{n}\right), \gamma\left(t_{1}\right)=\gamma_{1}\left(t_{1}\right)$ and $\widehat{\gamma}(t)=\left(\gamma_{2}\left(t_{2}\right), \gamma_{3}\left(t_{3}\right), \ldots, \gamma_{n}\left(t_{n}\right)\right)$. Consider the surface

$$
\omega:=\left\{\gamma(s)+\widehat{\gamma}(t):(s, t) \in \partial\left([0,1] \times[0,1]^{n-1}\right)\right\} .
$$


Let $\delta=\delta\left(s_{1}, s_{2}\right)$ be the diameter of $\gamma\left(\left[s_{1}, s_{2}\right]\right)$ for $s_{1}, s_{2} \in[0,1]$. Clearly, $\delta \rightarrow 0$ as $s_{1} \rightarrow s_{2}$. Pick $s_{1}$ and $s_{2}$ sufficiently close so the diameter of $\gamma_{i}([0,1])$ is greater than $2 \delta$ for all $i$. Hence there exists a point on the surface $\gamma\left(s_{1}\right)+\widehat{\gamma}\left([0,1]^{n-1}\right)$ that is not in the $\delta$-neighbourhood of $\gamma\left(\left[s_{1}, s_{2}\right]\right)+$ $\gamma\left(\partial\left([0,1]^{n-1}\right)\right)$. By Lemma 1.4, either there exists a point in the interior of this surface, or $\gamma\left(s_{1}\right)+\widehat{\gamma}\left([0,1]^{n-1}\right)$ and $\gamma\left(s_{2}\right)+\widehat{\gamma}\left([0,1]^{n-1}\right)$ coincide outside the $\delta$-neighbourhood of $\gamma\left(\left[s_{1}, s_{2}\right]\right)+\widehat{\gamma}\left(\partial\left([0,1]^{n-1}\right)\right)$.

Taking $s_{1} \rightarrow s_{2}$ and assuming that there is never a point in the interior gives that $\widehat{\gamma}\left([0,1]^{n-1}\right)$ admits an arbitrarily small translation symmetry outside its endpoints. This in turn gives that $\widehat{\gamma}\left([0,1]^{n-1}\right)$ is a $n-1$ dimensional plane, and that $\gamma([0,1])$ lies within this plane. Hence $\gamma_{1}, \gamma_{2}, \ldots, \gamma_{n}$ do not span is $\mathbb{R}^{n}$, a contradiction.

We need two more results before we can get on with the proof of Theorem 1.1 .

Lemma 1.6. [11, Lemma 2.3] The set $A_{M}$ is connected if $|\operatorname{det} M| \geq \frac{1}{2}$.

Lemma 1.7. [10, Lemma 4.1] Let $Y$ be a topological space. Suppose $f$ : $\{m, p\}^{\mathbb{N}} \rightarrow Y$ is a continuous map such that

$$
f([w m]) \cap f([w p]) \neq \varnothing
$$

for all $w \in\{m, p\}^{*}$. (Here $m$ stands for -1 and $p$ for 1 .) Then the image of $f$ is path connected.

Here $\left[i_{1} \ldots i_{k}\right]$ is the cylinder $\left\{\left\{a_{j}\right\}_{j=1}^{\infty} \subset\{p, m\}^{\mathbb{N}} \mid a_{j}=i_{j}, j=1, \ldots, k\right\}$.

Using $f:=\pi_{M}$ and $Y=\mathbb{R}^{d}$, we see that $A_{M}$ is the image of $f$. This gives the following corollary.

Corollary 1.8. The set $A_{M}$ is path connected if $|\operatorname{det} M| \geq \frac{1}{2}$.

Proof of Theorem 1.1. Let us first change the set of "digits" for this particular proof. Namely, consider the affine change of coordinates $x \mapsto \frac{1}{2}\left(x+\sum_{k=0}^{\infty} M^{k} u\right)$; this change corresponds to $a_{k} \mapsto \frac{1}{2}\left(a_{k}+1\right) \in\{0,1\}$. Recall that $u$ is chosen 
to be a cyclic vector. Thus, we have

$$
\begin{aligned}
\widetilde{A}_{M} & =\left\{\widetilde{\pi}_{M}\left(a_{0} a_{1} \ldots\right) \mid a_{k} \in\{0,1\}\right\} \\
& =\left\{\sum_{k=0}^{\infty} a_{k} M^{k} u \mid a_{k} \in\{0,1\}\right\} \\
& =\left\{\sum_{n=0}^{\infty} \sum_{j=0}^{d-1} a_{d n+j} M^{d n+j} u \mid a_{d n+j} \in\{0,1\}\right\} \\
& =\left\{\sum_{j=0}^{d-1} M^{j} \sum_{n=0}^{\infty} a_{d n+j}\left(M^{d}\right)^{n} u \mid a_{d n+j} \in\{0,1\}\right\} \\
& =\widetilde{A}_{M^{d}}+M \cdot \widetilde{A}_{M^{d}}+\cdots+M^{d-1} \cdot \widetilde{A}_{M^{d}},
\end{aligned}
$$

where $M \cdot X=\{M x: x \in X\}$. Now, if $\left|\operatorname{det} M^{d}\right| \geq \frac{1}{2}$, then by Corollary 1.8, the attractor $\widetilde{A}_{M^{d}}$ is path connected. We have $u=\widetilde{\pi}_{M^{d}}(1000 \ldots)$, whence $u \in \widetilde{A}_{M^{d}}$. Notice that

$$
\operatorname{span}\left\{M^{n} u \mid n \geq 0\right\}=\operatorname{span}\left\{M^{n} u \mid 0 \leq n \leq d-1\right\}=\mathbb{R}^{d},
$$

since $M^{n}$ is a linear combination of $I, M, \ldots, M^{d-1}$ for all $n \geq d$, in view of the Cayley-Hamilton theorem.

Choose now any path $\gamma$ in $\widetilde{A}_{M^{d}}$ which contains $u$. By (1.1), the paths $\gamma, M \gamma, \ldots, M^{d-1} \gamma$ span $\mathbb{R}^{d}$ as well, whence by Proposition 1.5, $\widetilde{A}_{M}$ has nonempty interior, and thus, so does $A_{M}$.

Remark 1.9. For $d=2$, Theorem 1.1 implies that if both eigenvalues of $M$ are greater than or equal to $2^{-1 / 4} \approx 0.8409$ in modulus, then $A_{M}$ has nonempty interior. This is essentially [6, Theorem 1.1]. Notice, however, that for $M$ having real eigenvalues in [6, Theorem 1.1] contains better bounds, due to a different proof. In particular, if $M=\left(\begin{array}{cc}-\lambda & 0 \\ 0 & \mu\end{array}\right)$ with $0<\lambda \leq \mu<1$, then we have the same claim with $\lambda \geq 2^{-1 / 2} \approx 0.7071$, and this bound is sharp if $\lambda=\mu$.

\section{ThE SET OF UNIQUENESS}

Let $\mathcal{U}_{M}$ be the set of uniqueness for our IFS, i.e., the set of $x \in A_{M}$ each of which has a unique address. We let $U_{M}$ denote the set of unique addresses for $A_{M}$, so $\mathcal{U}_{M}=\pi_{M}\left(U_{M}\right)$. For $d=1$ the set of uniqueness is a well studied topic - see, e.g., [9] and references therein.

When $d=2$, the following result holds:

Theorem 2.1. Let $M$ be a contractive $2 \times 2$ matrix which we assume to be - after an appropriate change of coordinates - one of the following: 
(1) $M=\left(\begin{array}{ll}\lambda & 1 \\ 0 & \lambda\end{array}\right)$. For any $\lambda \neq 0$, the set of uniqueness has positive Hausdorff dimension. [6, Corollary 4.8].

(2) $M=\left(\begin{array}{cc}\lambda_{1} & 0 \\ 0 & \lambda_{2}\end{array}\right)$. For any $0<\lambda_{1}<\lambda_{2}<1$, the set of uniqueness has positive Hausdorff dimension. [5, Corollary 4.3].

(3) $M=\left(\begin{array}{cc}\lambda_{1} & 0 \\ 0 & \lambda_{2}\end{array}\right)$. For any $-1<\lambda_{1}<0<\lambda_{2}<1$ with $\left|\lambda_{1}\right| \neq\left|\lambda_{2}\right|$, the set of uniqueness has positive Hausdorff dimension. [6, Corollary $4.5]$.

(4) $M=\left(\begin{array}{cc}a & b \\ -b & a\end{array}\right)$ with $\kappa=a+b i$. For any $\kappa$ with $\arg (\kappa) / \pi \notin \mathbb{Q}$ the set of uniqueness has positive Hausdorff dimension. [6, Section 4.3.1].

(5) $M=\left(\begin{array}{cc}a & b \\ -b & a\end{array}\right)$ with $\kappa=a+b i$. For any $\kappa$ with $\arg (\kappa) / \pi \in \mathbb{Q}$ set $q>0$ minimal such that $\kappa^{q} \in \mathbb{R}$ and let $\beta=|\kappa|^{-q}$. Then the set of uniqueness $\mathcal{U}_{M}$ is as follows:

(a) finite non-empty if $\beta \in(1, G]$;

(b) infinite countable for $\beta \in\left(G, \beta_{*}\right)$;

(c) an uncountable set of zero Hausdorff dimension if $\beta=\beta_{*}$; and

(d) a set of positive Hausdorff dimension for $\beta \in\left(\beta_{*}, \infty\right)$.

[6. Theorem 4.16].

(6) $M=\left(\begin{array}{cc}-\lambda & 0 \\ 0 & \lambda\end{array}\right)$ with $0<\lambda<1$. Then we have the same claim as in the previous item with $\beta=\lambda^{-2}$. [6, Proposition 4.21]

Here $G=\frac{1+\sqrt{5}}{2}$ and $\beta_{*} \approx 1.7872$ is the Komornik-Loreti constant introduced in [8]. The Komornik-Loreti constant is defined as the unique solution of the equation $\sum_{n=1}^{\infty} \mathfrak{m}_{n} x^{-n+1}=1$, where $\mathfrak{m}=\left(\mathfrak{m}_{n}\right)_{1}^{\infty}$ is the Thue-Morse sequence

$$
\mathfrak{m}=011010011001011010010110 \ldots,
$$

i.e., the fixed point of the substitution $0 \rightarrow 01,1 \rightarrow 10$.

The following result is straightforward.

Lemma 2.2. Let $M$ be a block matrix, i.e.,

$$
M=\left(\begin{array}{cc}
M_{1} & 0 \\
0 & M_{2}
\end{array}\right) .
$$

Then $U_{M} \supset U_{M_{j}}$ for $j \in\{1,2\}$.

Proof. Notice that

$$
\pi_{M}\left(a_{0} a_{1} a_{2} \ldots\right)=\left(\begin{array}{c}
\pi_{M_{1}}\left(a_{0} a_{1} \ldots\right) \\
\pi_{M_{2}}\left(a_{0} a_{1} \ldots\right)
\end{array}\right)
$$


We see that if one of the two coordinates on the right hand side is unique, then the left hand side must also be unique.

Corollary 2.3. If $\operatorname{dim}_{H} \mathcal{U}_{M_{1}}>0$ or $\operatorname{dim}_{H} \mathcal{U}_{M_{2}}>0$, then $\operatorname{dim}_{H} \mathcal{U}_{M}>0$.

Remark 2.4. Note that this claim is not if and only if. To see this, take, for instance, $M_{1}$ and $M_{2}$ both $1 \times 1$ real matrices with positive eigenvalues $\lambda \in\left(\frac{\sqrt{5}-1}{2}, 1\right)$ and $\mu \in\left(\frac{\sqrt{5}-1}{2}, 1\right)$ with $\lambda \neq \mu$. Then $\operatorname{dim}_{H} \mathcal{U}_{M}>0([5$, Corollary 4.3]), whereas $\mathcal{U}_{M_{1}}$ and $\mathcal{U}_{M_{2}}$ are finite - see [2].

By converting a matrix $M$ to Jordan normal form, this gives a rich family of matrices for which $\operatorname{dim}_{H} \mathcal{U}_{M}>0$. In particular, this allows us to prove

Theorem 2.5. Let $M$ be a $d \times d$ matrix.

(1) If $M$ has a non-trivial Jordan block, then $\operatorname{dim}_{H} \mathcal{U}_{M}>0$.

(2) If $M$ has an eigenvalue $\kappa$ with $\arg (\kappa) / \pi \notin \mathbb{Q}$, then $\operatorname{dim}_{H} \mathcal{U}_{M}>0$.

(3) If $M$ has two eigenvalues $\kappa_{1}$ and $\kappa_{2}$ with $\left|\kappa_{1}\right| \neq\left|\kappa_{2}\right|$, then $\operatorname{dim}_{H} \mathcal{U}_{M}>$ 0 .

(4) Let $M$ have only distinct simple eigenvalues, $\kappa_{1}, \kappa_{2}, \ldots, \kappa_{d}$ with $\arg \left(\kappa_{j}\right) / \pi \in$ $\mathbb{Q}$ for all $j$. Assume further $\left|\kappa_{1}\right|=\cdots=\left|\kappa_{d}\right|$. Let $q \in \mathbb{N}$ be minimal such that $\kappa_{j}^{q} \in \mathbb{R}, 1 \leq j \leq d$. If there exists $j$ and $k$ such that $\kappa_{j}^{q} \kappa_{k}^{q}<0$, then put $\beta=\left|\kappa_{1}\right|^{-2 q}$, otherwise put $\beta=\left|\kappa_{1}\right|^{-q}$. Then the set of uniqueness $\mathcal{U}_{M}$ is as follows:

(a) finite non-empty if $\beta \in(1, G]$;

(b) infinite countable for $\beta \in\left(G, \beta_{*}\right)$;

(c) an uncountable set of zero Hausdorff dimension if $\beta=\beta_{*}$; and

(d) a set of positive Hausdorff dimension for $\beta \in\left(\beta_{*}, \infty\right)$.

Some of these follow directly from Theorem 2.1 and Lemma 2.2. In Section 3 we show the case of Jordan blocks of size greater than or equal to 3 , and Jordan blocks of complex eigenvalues. That is, we show Theorem 2.5 (11). Theorem 2.5 (2) follows directly from Theorem 2.1 and Lemma 2.2. In Section 4 we prove cases (3) and (4).

There is a natural correspondence between a $2 \times 2$ real matrix $\left(\begin{array}{cc}a & b \\ -b & a\end{array}\right)$ and the $1 \times 1$ complex matrix $(a+b i)$. For notational reasons, we will often use this second form for a matrix or sub-matrix corresponding to a complex eigenvalue of $M$. 


\section{JORDAN BLOCKS}

Lemma 3.1. Let

$$
M=\left(\begin{array}{ccccc}
\kappa & 1 & & & 0 \\
& \kappa & 1 & & \\
& & \ddots & \ddots & \\
& & & \kappa & 1 \\
0 & & & & \kappa
\end{array}\right)
$$

with $0<|\kappa|<1$. Then $\operatorname{dim}_{H} \mathcal{U}_{M}>0$.

Proof. First, assume that $\kappa \in \mathbb{R}$. Let

$$
M^{\prime}=\left(\begin{array}{cc}
\kappa & 1 \\
0 & \kappa
\end{array}\right) \text {. }
$$

By [6, Lemma 3.1], we have:

$$
\pi_{M}\left(a_{0} a_{1} a_{2} \ldots\right)=\left(\begin{array}{c}
\frac{1}{(k-1) !} \frac{d^{k-1}}{\frac{1}{\kappa^{k-1}} \sum_{j=0}^{\infty} a_{j} \kappa^{j}} \\
\frac{1}{(k-2) !} \frac{d^{k-2}}{d \kappa^{k-2}} \sum_{j=0}^{\infty} a_{j} \kappa^{j} \\
\vdots \\
\frac{d}{d \kappa} \sum_{j=0}^{\infty} a_{j} \kappa^{j} \\
\sum_{j=0}^{\infty} a_{j} \kappa^{j}
\end{array}\right)
$$

and for $M^{\prime}$ we have

$$
\pi_{M^{\prime}}\left(a_{0} a_{1} a_{2} \ldots\right)=\left(\begin{array}{c}
\frac{d}{d \kappa} \sum_{j=0}^{\infty} a_{j} \kappa^{j} \\
\sum_{j=0}^{\infty} a_{j} \kappa^{j}
\end{array}\right) .
$$

(Here we are assuming $u$ our cyclic vector is $\left(\begin{array}{llll}0 & \ldots & 0 & 1\end{array}\right)^{T}$.) Hence if $a_{0} a_{1} \cdots \in U_{M^{\prime}}$, then the last two coordinates of $\pi_{M}\left(a_{0} a_{1} \ldots\right)$ form a unique pair, whence $a_{0} a_{1} \cdots \in U_{M}$. As $\operatorname{dim}_{H} \mathcal{U}_{M^{\prime}}>0$ from [6, Corollary 4.8], the result follows.

Next assume that $\kappa \notin \mathbb{R}$. If $\arg (\kappa) / \pi \notin \mathbb{Q}$, then we can repeat the above proof with $M^{\prime}=(\kappa)$ and [6, Section 4.3.1]. So assume that $\arg (\kappa / \pi) \in \mathbb{Q}$. From the techniques above, we see that it suffices to show the $2 \times 2$ case, after which the result will follow. Let

$$
M=\left(\begin{array}{cc}
\kappa & 1 \\
0 & \kappa
\end{array}\right)
$$

with $0<|\kappa|<1, \arg (\kappa) / \pi \in \mathbb{Q}$. Let $\left(z_{1}, z_{2}\right) \in A_{M}$ with $z_{1}, z_{2} \in \mathbb{C}$.

Let $q>0$ be minimal such that $\kappa^{q} \in \mathbb{R}$. Let

$$
M^{\prime}=\left(\begin{array}{cc}
\kappa^{q} & 1 \\
0 & \kappa^{q}
\end{array}\right)
$$

and consider the set $F=\left\{\left(a_{0} a_{1} a_{2} a_{3} a_{4} \ldots\right)\right\}$ where

$$
a_{j}=\left\{\begin{array}{ll}
-1 & \text { if } \Im\left(\kappa^{j}\right)<0 \\
+1 & \text { if } \Im\left(\kappa^{j}\right)>0 \\
-1 \text { or }+1 & \text { if } \Im\left(\kappa^{j}\right)=0
\end{array} \quad \text { for all } j\right.
$$


and let $\mathcal{F}=\pi_{M}(F)$. We note that $\Im\left(\kappa^{j}\right)=0$ if and only if $q \mid j$.

Let $s=\max \left(\Im\left(z_{2}\right):\left(z_{1}, z_{2}\right) \in A_{M}\right)$. We see that $\left(z_{1}, z_{2}\right) \in \mathcal{F}$ if and only if $\Im\left(z_{2}\right)=s$. Furthermore, we see that there is a map $\varphi$ from $\{ \pm 1\}^{\mathbb{N}}$ to $\mathcal{F}$ given by

$$
\varphi\left(b_{0} b_{1} b_{2} \ldots\right)=\pi_{M}\left(b_{0} a_{1} a_{2} \ldots a_{q-1} b_{1} a_{q+1} \ldots\right)
$$

where $a_{1}, a_{2}, a_{3}$, etc are chosen to as above. The map $\varphi$ is one-to-one, and moreover, it is clearly Hölder continuous in the standard metric. This gives us that if a point is unique in $A_{M^{\prime}}$, then the corresponding point in $\mathcal{F}$ is unique, from which the result follows.

\section{Complex eigenvalues}

Lemma 4.1. Let $\kappa_{1}, \kappa_{2}, \ldots, \kappa_{d}$ be such that $\arg \left(\kappa_{j}\right) / \pi \in \mathbb{Q}$. Let $q>0$ be minimal such that $\kappa_{j}^{q} \in \mathbb{R}$ for all $j$.

$$
M=\left(\begin{array}{cccc}
\kappa_{1} & & & 0 \\
& \kappa_{2} & & \\
& & \ddots & \\
0 & & & \kappa_{d}
\end{array}\right), M^{\prime}=\left(\begin{array}{cccc}
\kappa_{1}^{q} & & & 0 \\
& \kappa_{2}^{q} & & \\
& & \ddots & \\
0 & & & \kappa_{d}^{q}
\end{array}\right) \text {. }
$$

We have $\operatorname{dim}_{H}\left(\mathcal{U}_{M^{\prime}}\right)>0$ if and only if $\operatorname{dim}_{H}\left(\mathcal{U}_{M}\right)>0$.

Proof. Similarly to the proof of the complex part of Lemma 3.1, consider the set $F=\left\{\left(a_{0} a_{1} a_{2} a_{3} a_{4} \ldots\right)\right\}$ where

$a_{j}= \begin{cases}-1 & \text { if } \Im\left(\kappa_{1}^{j}\right)<0 \\ +1 & \text { if } \Im\left(\kappa_{1}^{j}\right)>0 \\ -1 & \text { if } \Im\left(\kappa_{1}^{j}\right)=0 \text { and } \Im\left(\kappa_{2}^{j}\right)<0 \\ +1 & \text { if } \Im\left(\kappa_{1}^{j}\right)=0 \text { and } \Im\left(\kappa_{2}^{j}\right)>0 \\ \vdots & \vdots \\ -1 & \text { if } \Im\left(\kappa_{1}^{j}\right)=\Im\left(\kappa_{2}^{j}\right)=\cdots=\Im\left(\kappa_{n}^{j}\right)=0 \text { and } \Im\left(\kappa_{n+1}^{j}\right)<0 \\ +1 & \text { if } \Im\left(\kappa_{1}^{j}\right)=\Im\left(\kappa_{2}^{j}\right)=\cdots=\Im\left(\kappa_{n}^{j}\right)=0 \text { and } \Im\left(\kappa_{n+1}^{j}\right)>0 \\ \vdots & \vdots \\ -1 \text { or }+1 & \text { if } \Im\left(\kappa_{1}^{j}\right)=\Im\left(\kappa_{2}^{j}\right)=\cdots=\Im\left(\kappa_{d}^{j}\right)=0\end{cases}$

for all $j$ and let $\mathcal{F}=\pi(F)$. We note that $\Im\left(\kappa_{1}^{j}\right)=\cdots=\Im\left(\kappa_{d}^{j}\right)=0$ if and only if $q \mid j$. 
Put

$$
\begin{aligned}
& s_{1}=\max \left(\Im\left(z_{1}\right):\left(z_{1}, \ldots, z_{d}\right) \in A\right), \\
& s_{2}=\max \left(\Im\left(z_{2}\right):\left(z_{1}, \ldots, z_{d}\right) \in A, \Im\left(z_{1}\right)=s_{1}\right), \\
& s_{3}=\max \left(\Im\left(z_{3}\right):\left(z_{1}, \ldots, z_{d}\right) \in A, \Im\left(z_{1}\right)=s_{1}, \Im\left(z_{2}\right)=s_{2}\right), \\
& \quad \vdots \\
& s_{d}=\max \left(\Im\left(z_{d}\right):\left(z_{1}, \ldots, z_{d}\right) \in A, \Im\left(z_{1}\right)=s_{1}, \Im\left(z_{2}\right)=s_{2}, \ldots, \Im\left(z_{d-1}\right)=s_{d-1}\right) .
\end{aligned}
$$

We see that $\left(z_{1}, z_{2}, \ldots, z_{d}\right) \in \mathcal{F}$ if and only if $\Im\left(z_{j}\right)=s_{j}$ for $j=1,2, \ldots, d$. Furthermore, the map $\psi:\{ \pm 1\}^{\mathbb{N}} \rightarrow \mathcal{F}$ defined by

$$
\psi\left(b_{0} b_{1} \ldots\right)=\pi_{M}\left(b_{0} a_{1} a_{2} \ldots a_{q-1} b_{1} a_{k+1} a_{k+2} \ldots\right),
$$

where the $a_{1}, a_{2}, a_{3}$, etc are chosen as above, is one-to-one and Hölder continuous. This gives us that if a point is unique in $A_{M^{\prime}}$, then the corresponding point in $\mathcal{F}$ is unique. Moreover, $\operatorname{dim}_{H} \mathcal{U}_{M^{\prime}}>0$ implies $\operatorname{dim}_{H} \mathcal{U}_{M}>0$.

For the other direction, assume that that $x=\pi_{M}\left(a_{0} a_{1} a_{2} \ldots\right)$ is in $\mathcal{U}_{M}$. Consider the point $\pi_{M^{\prime}}\left(a_{0} a_{q} a_{2 q} \ldots\right) \in A_{M^{\prime}}$. If it is not a point of uniqueness, then there exists a $\pi_{M^{\prime}}\left(b_{0} b_{q} b_{2 q} \ldots\right)=\pi_{M^{\prime}}\left(a_{0} a_{q} a_{2 q} \ldots\right)$. But by construction $x=\pi_{M}\left(b_{0} a_{1} a_{2} \ldots a_{q-1} b_{q} a_{q+1} \ldots\right)$, a contradiction.

A similar argument can be used for the subsequence $a_{j} a_{q+j} a_{2 q+j} \ldots$ mapping to a simple linear transformation of $\mathcal{U}_{M^{\prime}}$, namely, $M^{j} \mathcal{U}_{M^{\prime}}$.

Hence for any point of uniqueness in $\mathcal{U}_{M}$ we have $q$ maps into affine copies of $\mathcal{U}_{M^{\prime}}$, each one giving a point of uniqueness. If $\operatorname{dim}_{H} \mathcal{U}_{M}>0$, then one of these maps will also have have positive Hausdorff dimension, from which the result follows.

Now we are ready to conclude the proof of Theorem 2.5. Note first that if $\left|\kappa_{1}\right| \neq\left|\kappa_{2}\right|$, then $\left|\kappa_{1}^{q}\right| \neq\left|\kappa_{2}^{q}\right|$ with $\kappa_{1}^{q}, \kappa_{2}^{q} \in \mathbb{R}$. From this Theorem 2.5 (3) follows from Theorem 2.1 (2) or (3).

If $\left|\kappa_{1}\right|=\cdots=\left|\kappa_{d}\right|=\lambda$, then $M^{\prime}=\lambda^{q} J$, where $J$ is a $d \times d$ diagonal matrix with -1 or 1 on the diagonal. If there exists $j$ and $k$ such that $\kappa_{j}^{q} \kappa_{k}^{q}<0$, then $J$ will contain both a -1 and a 1 , and this will follow from Theorem 2.1 (6) . If no such $j$ and $k$ exists, then the result follows from [3, Theorem 2].

\section{REFERENCES}

[1] K. Dajani, K. Jiang and T. Kempton, Self-affine sets with positive Lebesgue measure, Indag. Math. 25 (2014), 774-784.

[2] P. Erdős, I. Joó and V. Komornik, Characterization of the unique expansions $1=$ $\sum_{i=1}^{\infty} q^{-n_{i}}$ and related problems, Bull. Soc. Math. Fr. 188 (1990), 377-390. 
[3] P. Glendinning and N. Sidorov, Unique representations of real numbers in non-integer bases, Math. Res. Lett. 8 (2001), 535-543.

[4] C. S. Güntürk, Simultaneous and hybrid beta-encodings, in Information Sciences and Systems, 2008. CISS 2008. 42nd Annual Conference on, pages 743-748, 2008.

[5] K. G. Hare and N. Sidorov, On a family of self-affine sets: topology, uniqueness, simultaneous expansions, Ergod. Th. Dynam. Sys., published electronically, doi:10.1088/0951-7715/29/1/1.

[6] K. G. Hare and N. Sidorov, Two-dimensional self-affine sets with interior points, and the set of uniqueness, Nonlinearity 29 (2016), 1-26.

[7] V. Kleptsyn, The Minkowski sum of two curves, http://mathoverflow.net/questions/194350/the-minkowski-sum-of-two-curves

[8] V. Komornik and P. Loreti, Unique developments in non-integer bases, Amer. Math. Monthly 105 (1998), 636-639.

[9] V. Komornik and M. de Vries, Unique expansions of real numbers, Adv. Math. 221 (2009), 390-427.

[10] M. Odlyzko and B. Poonen, Zeros of polynomials with 0,1 coefficients, Enseign. Math. (2), 39 (1993), 317-348.

[11] P. Shmerkin and B. Solomyak, Zeros of $\{-1,0,1\}$ power series and connectedness loci for self-affine sets, Exp. Math. 15 (2006), 499-511.

Department of Pure Mathematics, University of Waterloo, Waterloo, Ontario, Canada N2L 3G1

E-mail address: kghare@uwaterloo.ca

School of Mathematics, The University of Manchester, Oxford Road, MAnChester M13 9PL, United Kingdom.

E-mail address: sidorov@manchester.ac.uk 